subjects with 1, 2 and 3 positive samples during a 24 hour period were $52 \%, 14 \%$ and $14 \%$ respectively in IPF patients and 20\%, $12 \%$ and $4 \%$ in control subjects. There was no significant difference in reflux-related quality of life or respiratory quality of life between pepsin positive and pepsin negative patients measured using the REFLUX questionnaire (mean 93.6 \pm 2.6 SEM vs $97.8 \pm 2.3, \mathrm{p}=0.47)$ and SGRQ $(49.5 \pm 3.5$ vs $34 \pm 11.9$, $\mathrm{p}=0.1)$. The HARQ score was significantly higher in pepsin positive patients $(23.8 \pm 3.3$ vs $7.5 \pm 3.3, \mathrm{p}=0.03)$.

Conclusion Salivary pepsin measurement is simple, convenient and acceptable to patients. Our results confirm an increased prevalence of positive salivary pepsin in IPF patients compared to healthy volunteers but demonstrate a marked temporal variability. Therefore, more than one sample or repeated sample collection is required for optimal sensitivity.

\section{S31 PEPSIN DETECTION DESPITE THE USE OF ACID SUPPRESSANT MEDICATION IN PATIENTS WITH AIRWAY REFLUX RELATED CHRONIC COUGH}

${ }^{1} \mathrm{GL}$ Crossfield, ${ }^{2} \mathrm{~W}$ Jackson, ${ }^{2} \mathrm{~J}$ Burke, ${ }^{3} \mathrm{AD}$ Woodcock, ${ }^{3} \mathrm{~V}$ Strugala, ${ }^{4} \mathrm{C}$ Ward, ${ }^{1} \mathrm{JP}$ Pearson, ${ }^{3} \mathrm{PW}$ Dettmar, ${ }^{5} \mathrm{AH}$ Morice; ${ }^{1}$ Institute of Cell and Molecular Biosciences, Newcastle Upon Tyne, United Kingdom; ${ }^{2}$ Gastro-Intestinal Physiology Laboratory, Hull, United Kingdom; ${ }^{3}$ Technostics Ltd, Hull, United Kingdom; ${ }^{4}$ Institute of Cellular Medicine, Newcastle Upon Tyne, United Kingdom; ${ }^{5}$ Cardiovascular and Respiratory Studies, East Yorkshire, United Kingdom

\subsection{6/thoraxjn-2013-204457.38}

Background Chronic cough (CC) is an increasing problem that is not easy to treat with medication. Associated symptoms include hoarse voice, dysphonia, persistent tickling and irritation of the throat or chest. These lead to poor sleeping and eating patterns, loss of vocal independence and social isolation all resulting in an impaired quality of life. Airway reflux is a common cause of unexplained chronic cough and proton pump inhibitor (PPI) medication is commonly prescribed as initial therapy. The following study assessed pepsin identification in CC patients as a marker of airway reflux on PPI.

Methods Symptomatic expectorated saliva samples were obtained from 16 patients (6 male/10 female, 50 years (37-76), Body Mass Index (BMI) 30 (24-44), median (range)) attending clinical appointment with symptoms of chronic cough. Pepsin was identified using the Peptest ${ }^{\mathrm{TM}}$ an in vitro diagnostic medical device specific for human pepsin A (RD Biomed Ltd, UK). All patients completed the Hull Airways Reflux Questionnaire (HARQ) to determine airway reflux related cough (range $0-70 ;<13$ normal). Patient demographics and medication data was provided on sample collection. Results Fourteen (88\%) of the CC patients were positive for pepsin in saliva samples (median $83 \mathrm{ng} / \mathrm{ml}$; range $25-250$ ), providing non-invasive verification of presence of reflux in this CC population. Thirteen pepsin positive patients were symptomatic of airway reflux related cough according to abnormal HARQ score (median 40; range 25-59) and all were taking PPI (20-60mg/d range collected from referral letter and patient questionnaire). The median BMI of the pepsin positive patients was 30 (range 25-44). Conclusion Pepsin was present in $88 \%$ of suspected airway reflux related chronic cough patients therefore corroborating the diagnosis of reflux. Airway reflux is associated with unexplained chronic cough in patients receiving PPI highlighting that symptoms and reflux are still present despite acid suppression. Overweight and obese BMI status is a common feature of airway reflux related chronic cough patients. A reconsideration of the empiric use of acid suppression use maybe warranted for unexplained chronic cough.

\section{S32 PEPSIN DETECTION IN EXPECTORATED SALIVA: A USEFUL MARKER FOR AIRWAY REFLUX?}

${ }^{1} \mathrm{~S}$ Faruqi, ${ }^{2} \mathrm{AD}$ Woodcock, ${ }^{2} \mathrm{PW}$ Dettmar, ${ }^{1} \mathrm{AH}$ Morice; ${ }^{1}$ Division of Cardiovascular and Respiratory Studies, University of Hull, Castle Hill Hospital, Cottingham, UK; ${ }^{2}$ RD Biomed Ltd, Castle Hill Hospital, Cottingham, UK

\subsection{6/thoraxjnl-2013-204457.39}

Introduction Gastro oesophageal reflux (GOR) is a very common cause for chronic cough. The clinical history of airway reflux differs from that of GOR disease and often the diagnosis of airways reflux is not considered. Although oesophageal investigations can support the diagnosis these are invasive, time consuming and expensive. The presence of pepsin in the oesophagus, or more proximally in the pharynx or the airways, suggest GOR. The aim of this study was to study the diagnostic utility of measuring pepsin in expectorated saliva in unselected patients presenting with chronic cough.

Methods Consecutive patients referred to the Hull Cough Clinic were instructed to collect expectorated saliva on three occasions following symptoms (paroxysm of cough). Saliva was collected into tubes containing $0.5 \mathrm{ml}$ of $0.01 \mathrm{M}$ citric acid and analysed for the presence of pepsin using a lateral flow test comprising two unique human monoclonal antibodies to pepsin (Peptest $^{\mathrm{TM}}$, RDBiomed Ltd). The cut off value to determine pepsin positivity was $25 \mathrm{ng} /$ ml. Patients also completed the Hull Airways Reflux Questionnaire (HARQ), a validated tool to diagnose airways reflux.

Results 72 patients were included in this study (females 49, mean age 58.3 years). Salivary pepsin assay was positive in at least one sample in 46 (64\%). 24, 10 and 12 patients had 1, 2 and 3 positive tests respectively. 10 samples had pepsin levels above $250 \mathrm{ng} /$ $\mathrm{ml}$. For purpose of comparison this data was examined against 300 similar pepsin assays from 100 healthy subjects with no typical or atypical reflux symptoms. In this group only 6 of 300 samples had more than $250 \mathrm{ng} / \mathrm{ml}$ of pepsin measured and $64 \%$ had all three samples negative for pepsin. ${ }^{1}$ This is shown in table 1 . The median HARQ score was 30 (range 1-67).

Conclusion A high proportion of patients with chronic cough have demonstrable levels of pepsin in expectorated saliva at the time of having symptoms. This non invasive test may be a useful investigation to support the diagnosis of airway reflux.

\begin{tabular}{|c|c|c|c|c|c|c|}
\hline & $\begin{array}{l}\text { At least one } \\
\text { sample positive }\end{array}$ & $\begin{array}{l}\text { One sample } \\
\text { positive }\end{array}$ & $\begin{array}{l}\text { Two samples } \\
\text { positive }\end{array}$ & $\begin{array}{l}\text { All three } \\
\text { samples positive }\end{array}$ & $\begin{array}{l}\text { All three } \\
\text { samples negative }\end{array}$ & $\begin{array}{l}\text { Pepsin level } \\
>250 \mathrm{ng} / \mathrm{ml}\end{array}$ \\
\hline $\begin{array}{l}\text { Chronic } \\
\text { cough } \\
\text { patients }\end{array}$ & $63.9 \%$ & $33.3 \%$ & $13.9 \%$ & $16.7 \%$ & $36.1 \%$ & $4.6 \%$ \\
\hline $\begin{array}{l}\text { Healthy } \\
\text { volunteers }\end{array}$ & $36 \%$ & $20 \%$ & $12 \%$ & $4 \%$ & $64 \%$ & $2 \%$ \\
\hline
\end{tabular}




\section{REFERENCES}

1. Hayat JO et al. Is pepsin detected in the saliva of healthy individuals? Gut 2013: 62 (Suppl 1) A108-A109.

\section{S33 DOES ANTI-REFLUX SURGERY SYMPTOMATICALLY IMPROVE EXTRA-OESOPHAGEAL SYMPTOMS AND QUALITY OF LIFE IN GASTRO-OESOPHAGEAL REFLUX DISEASE}

C A Lynch, S Ejiofor, R Sathyamurthy, A Mansur; Birmingham Heartlands Hospital, Birmingham, United Kingdom

\subsection{6/thoraxjnl-2013-204457.40}

Introduction Extra-oesophageal (ie laryngeal, pharyngeal and pulmonary) symptoms of gastro-oesophageal reflux (GOR) are common clinical problems. GOR can cause a chronic cough and its prevalence is higher in asthmatics than in the general population. Proton pump inhibitors (PPI) are the most effective available therapy but in those who remain symptomatic despite optimal medical therapy anti-reflux surgery is considered.

Method At our central England teaching hospital a database was set up for all respiratory patients with GOR who were referred for anti-reflux surgery. Symptoms were assessed at baseline (presurgery), 3 months post surgery and 12 months post surgery using the asthma quality of life questionnaire (AQLQ, range; $0=$ worst $-7=$ best) and a cough symptom questionnaire (range; $0=$ no cough -10 worst cough).

Results A total of 61 patients ( $70 \%$ females) with mean age of 48.64 (range $20-78$ years) were analysed. 34 patients were asthmatics and 26 patients had a chronic cough. 1 patient was excluded as they had vocal cord dysfunction.

All patients had significant GOR confirmed by oesophageal manometry and $\mathrm{pH}$ reflux studies [mean DeMeester score of $46.6(\mathrm{SD}=38.4$, normal range $<14.72$ ), hypotonic mean lower oesophageal sphincter pressure of $5.7 \mathrm{mmHg}$ (SD 3.78, normal range $=12-25)$ and a mean\% reflux time of 11.9 (SD 8.98, normal range <4\%)]. The baseline mean $\mathrm{FEV}_{1}$ in the asthma group was 2.08 (mean FEV1/FVC 0.72) compared to 2.61 (mean FEV1/FVC 0.81) in the cough group.

There was significant improvement in reflux symptoms. In the asthma group, the mean AQLQ score improved from baseline $(3.05, \mathrm{SD}=0.8)$ to 3 months post surgery $(3.68, \mathrm{SD}=0.9)$ $(\mathrm{p}=0.0235)$, and 12 months post surgery $(3.5, \mathrm{SD}=1.2)$ which did not reach statistical significance $(\mathrm{p}=0.1)$ [see Figure 1]. There was also marked improvement in the cough symptom score from baseline $(7.0, \mathrm{SD}=2.3)$ to 3 months post surgery $(2.8, \mathrm{SD}=3.1)(\mathrm{p}=<0.0001)$ [see Figure 1].

Conclusion In patients with evidence of severe GOR who remain symptomatic despite optimal medical management, anti-reflux surgery can improve cough and asthma related quality of life.
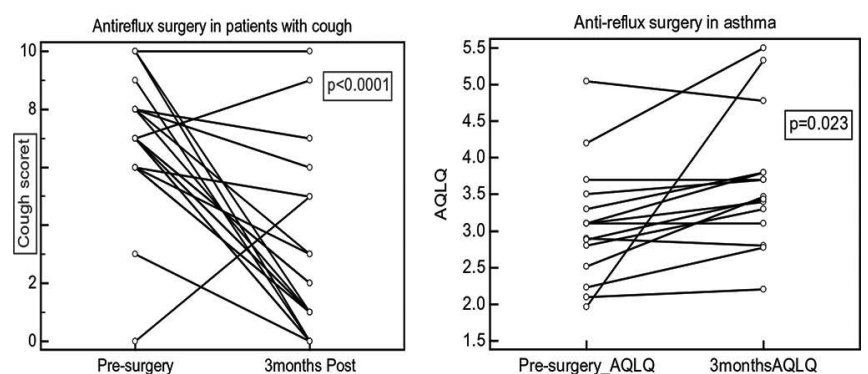

Abstract S33 Figure1 Does anti-reflux surgery symptomatically improve extra-oesophageal symptoms and quality of life in gastrooesophageal reflux disease
${ }^{1} \mathrm{R}$ Mackley, ${ }^{2} \mathrm{~T}$ Schatzberger, ${ }^{1} \mathrm{SM}$ Parker; ${ }^{1}$ Northumbria Healthcare NHS Foundation trust, North Shields, United Kingdom; ${ }^{2}$ Sele Medical Practice, Hexham, United Kingdom

\subsection{6/thoraxjnl-2013-204457.41}

Background Chronic cough is a common symptom in primary care. There appears to be significant variation in investigation and empirical treatment prior to referral on to secondary care. We looked at; a) Investigation/treatment undertaken prior to referral to secondary care and b) a survey of how GPs manage cough.

Method The study consisted of; a) Retrospective review of referrals (January 2012-January 2013) to a secondary care cough clinic and b) an online questionnaire on cough distributed to colleagues in primary care.

Results Of the primary care referrals, $47 / 58$ casenotes were available (mean age was 59(27-84), 63\% female). Only 4/47 $(8.7 \%)$ were current smokers. Median duration of symptoms at referral was 7 months (2-420), 35/47(74.5\%) had a chest X-Ray prior to referral, 18/47(39.1\%) spirometry, 3/47 had used ACE inhibitors. Most investigations performed prior to referral were normal. Empirical treatment attempted included; antireflux therapy $23 / 47(50 \%)$, inhaled corticosteroids $12 / 47(26.1 \%)$ and nasal steroids $9 / 47(19.6 \%)$. Various other treatments were used (including cough suppressants, antihistamines, leukotriene receptor antagonists and antibiotics) in 22/47(46\%).

Only 16/51(31\%) of questionnaire respondents were aware of published cough guidelines. Most respondents were aware of the definition of chronic cough (37/51(72.5\%)). When asked to list the 3 commonest causes of chronic cough, asthma was identified by $33(64.7 \%)$, GORD by $38(74.5 \%)$ and rhinitis/post-nasal drip by $20(39.2 \%)$. Treatments most commonly initiated included acid suppression (PPI's) 33/51(64.7\%), nasal spray 18/ $51(35.3 \%)$ and inhaled steroids $4 / 51(7.8 \%)$. The majority of patients do not get referred on to secondary care; 34/51(66.6\%) of respondents estimate they refer less than $10 \%$ of cases. Referral was usually triggered by the following factors - unclear diagnosis, failed treatment, patient concern or abnormal tests.

Conclusions Many patients are referred on to secondary care without basic investigations and appropriate trials of recommended therapy. Knowledge of chronic cough in primary care is limited and most GPs are unaware of published guidelines. Increased education and awareness of cough guidelines could improve management of cough in the community. This is a topic that requires further systematic study as there is very little research in this area.

\section{Cutting edge respiratory science}

\section{S35 MIF-CXCR4 AS A NOVEL AXIS FOR MESENCHYMAL STEM CELLS RECRUITMENT TO TUMOURS IN VIVO}

S Lourenco; Lungs for Living Research Centre, London, UK

10.1136/thoraxjnl-2013-204457.42

Mesenchymal Stem Cells (MSCs) are inherently tumour-homing, immunosuppressive and can be isolated, cultured, expanded, and transduced, making them viable candidates for cell therapy. MSCs can also be useful in allogeneic transplantation because of their immunocompatibility. MSCs have the capacity to home specifically to tumours including gliomas and breast, colon, ovarian, and lung carcinomas, among many other primary and metastatic tumours. Some discrepancies are however present regarding the mechanism and the involvement of molecules/receptors in MSC homing to tumours. 